\title{
Just the facts: point-of-care ultrasound for airway management
}

\author{
Michael Gottlieb ${ }^{1}$ (1) $\cdot$ Paul Olszynski ${ }^{2} \cdot$ Paul Atkinson $^{3}$
}

Received: 10 September 2020 / Accepted: 23 December 2020 / Published online: 25 January 2021

(C) The Author(s), under exclusive licence to Canadian Association of Emergency Physicians (CAEP)/ Association Canadienne de Médecine d'Urgence (ACMU) 2021

Keywords Ultrasound · PoCUS · Intubation · Airway · Cricothyroidotomy

\section{Clinical scenario}

A 72-year-old woman presents to the emergency department (ED) in cardiac arrest. She was intubated prior to arrival. The prehospital team states that the intubation was difficult and that end-tidal capnography did not demonstrate a good waveform. They are unclear if this is due to the endotracheal tube (ETT) location or poor perfusion. You wonder if the ETT is in the correct location.

\section{Key clinical questions}

\section{Why might PoCUS be useful during emergency airway management?}

There are several clinical maneuvers and devices used to help confirm ETT placement. Following direct visualization of the ETT passing the cords, clinicians may look for misting of the ETT, bilateral chest rise, and breath sounds. However, none of these are highly reliable nor are esophageal detector devices [1]. While waveform capnography is more reliable

Michael Gottlieb

michaelgottliebmd@gmail.com

Paul Olszynski

p.olszynski@usask.ca

Paul Atkinson

paul.atkinson@dal.ca

1 Department of Emergency Medicine, Rush University Medical Center, 1750 West Harrison Street, Suite 108 Kellogg, Chicago, IL 60612, USA

2 Department of Emergency Medicine, University of Saskatchewan, Saskatoon, SK, Canada

3 Department of Emergency Medicine, Dalhousie University, Saint John Regional Hospital, Saint John, NB, Canada than the aforementioned approaches, it can also be limited if the ETT is preceded by recent ingestion of carbonated beverages or when expired $\mathrm{CO}_{2}$ levels are low (e.g., massive pulmonary embolism, flash pulmonary edema, or cardiac arrest) [1]. In fact, capnography has a pooled sensitivity of only $60-88 \%$ in cardiac arrest patients [1-3]. On the other hand, point-of-care ultrasound (PoCUS) has been demonstrated to be $98.7 \%$ sensitive and $97.1 \%$ specific for confirming endotracheal intubation, with a positive likelihood ratio of 34.4 and negative likelihood ratio of 0.01 [2]. Moreover, PoCUS can rapidly confirm ETT placement (mean: $13.0 \mathrm{~s}$ ) and does not require positive pressure ventilations, which reduces the risk of gastric distension and potential aspiration [2]. Importantly, PoCUS is operator-dependent and relies on adequate training and skill maintenance.

\section{How do you perform PoCUS for intubation confirmation?}

Place a linear probe transversely on the suprasternal notch (Fig. 1) [1]. The trachea is located posterior to the thyroid gland and will appear as a hyperechoic (bright) circular structure with distal gray shadowing (Fig. 2). Assess for the presence of tracheal or esophageal intubation. An esophageal intubation will demonstrate a 'double tract' (or 'double lumen') sign, which will appear like a second, smaller 'trachea' just lateral to the true trachea (Fig. 2). This can be performed using either the static (post-intubation) or dynamic (assessed in real-time during the intubation attempt) technique. Studies have demonstrated no significant differences in diagnostic accuracy between the static and dynamic approaches [1, 2]. The static technique is beneficial because only one provider is needed (i.e., the intubating provider can also perform the post-intubation assessment). It also avoids having additional people near the patient's airway, as well as increased pressure on the patient's neck from the probe, which could make the intubation more challenging. 


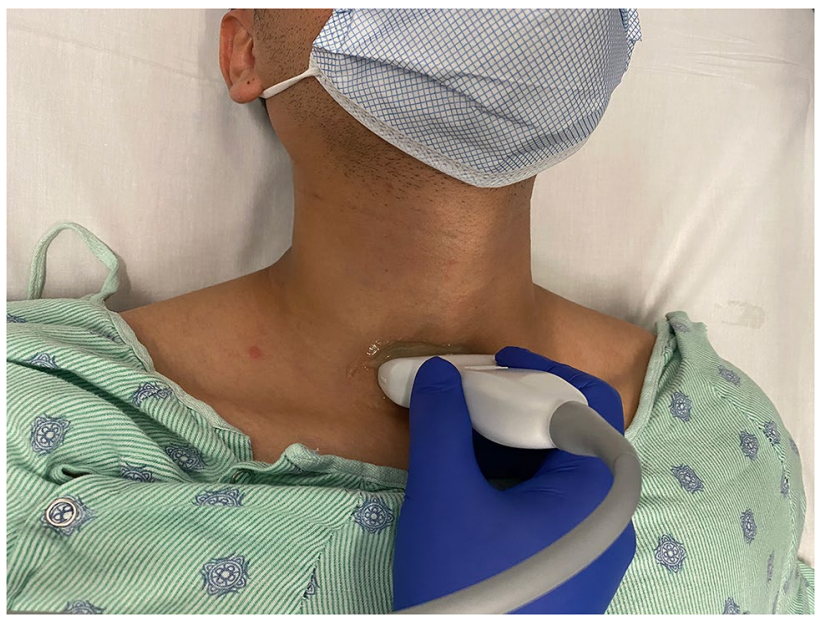

Fig. 1 Orientation of the probe for transtracheal ultrasound (indicator towards the patient's right side)

Alternatively, the dynamic technique may improve the ability to see the ETT as it passes through the trachea or esophagus by creating motion within these structures. One modification to improve visualization is by gently twisting the ETT side-to-side post-intubation to create a motion artifact [1]. We also recommend incorporating assessment for bilateral lung sliding or diaphragmatic elevation, which has been shown to improve accuracy compared with transtracheal PoCUS alone [1, 4]. As with other intubation confirmation methods, PoCUS is only a single tool and should be used in conjunction with other modalities (e.g., direct visualization, capnography).

\section{Can PoCUS be used to assess the depth of insertion for the endotracheal tube?}

Yes, PoCUS can also be utilized to assess the depth of the ETT. This can be assessed directly by visualizing the ETT cuff or indirectly using lung sliding. To directly visualize the ETT, place the probe in the transverse orientation in the suprasternal notch (similar to ETT confirmation) and assess for the presence of the ETT cuff after inflation [5]. It has been recommended to inject saline into the cuff to improve visualization [5]. One study reported that this technique was over $98 \%$ sensitive and over $96 \%$ specific for identifying the ETT depth [5]. Alternatively, and perhaps easier to perform, PoCUS can assess for the presence of lung sliding. The presence of bilateral lung sliding as the patient is ventilated, suggests that the ETT is sufficiently high to ventilate both lungs, while unilateral lung sliding is concerning for a right or left mainstem intubation [4]. Because unilateral absence of lung sliding can also be present with pneumothorax, it is important to also assess for the presence of a lung pulse. A lung pulse is the direct visualization of cardiac pulsations on lung ultrasound caused by cardiac contractions compressing an atelectatic lung [4]. This demonstrates that the visceral and parietal pleura remain opposed despite inadequate ventilation and can be used to help distinguish a pneumothorax (lung pulse absent) from a mainstem intubation (lung pulse present) [3].

\section{How can PoCUS be used for cricothyroidotomy?}

The landmark technique can be challenging. PoCUS has been demonstrated to be superior to the landmark technique and can be performed in under $30 \mathrm{~s}$ [1]. The transverse approach involves placing a linear probe transversely across the cricoid cartilage and advancing the probe cephalad until the thyroid cartilage is visualized. The thyroid cartilage will appear as a hyperechoic triangular structure. The transducer is then moved caudally to visualize the cricothyroid membrane, which will appear as a hyperechoic white line with reverberation artifact distally (Fig. 3). For the longitudinal technique, begin on the cricoid cartilage in the transverse orientation and rotate 90 degrees to the long axis. The tracheal rings will appear as a 'string of pearls'. Slide the probe superiorly to visualize the cricoid and tracheal cartilage (Fig. 3). After localizing the cricothyroid membrane in each plane, use a skin marker to mark the area to identify the midline (transverse technique) and level (longitudinal technique) of the cricothyroid membrane, thereby making a target on the neck. We suggest that PoCUS be used to mark the cricothyroid membrane location prior to the intubation attempt in all patients at a higher risk of difficult intubation once they are placed in position for intubation [6].
Fig. 2 Transtracheal ultrasound demonstrating a tracheal (left) and esophageal (right) intubation. $T$ Trachea, $E$ Esophagus (a)

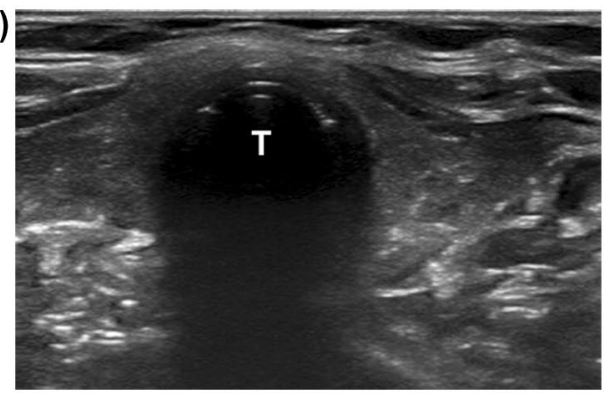

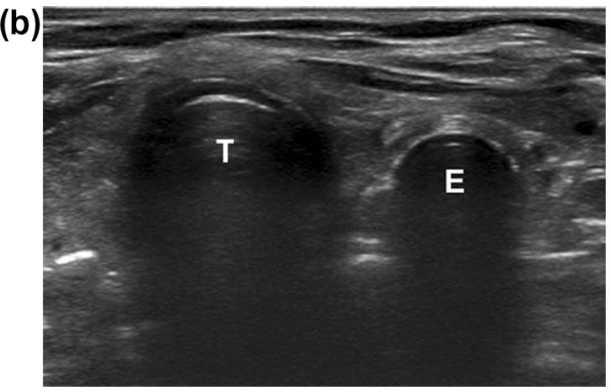


Fig. 3 Cricothyroid membrane (arrow) in the transverse (left) and longitudinal (right) plane (a)

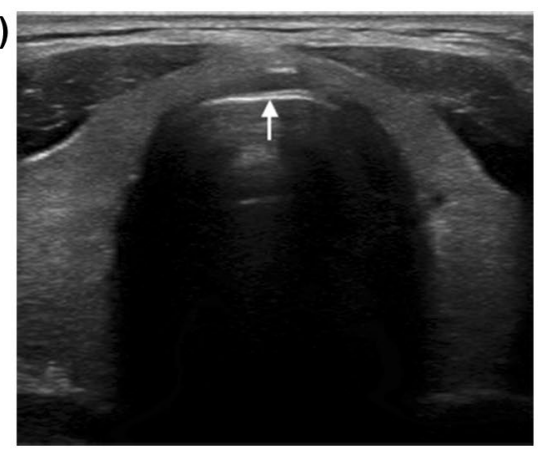

(b)

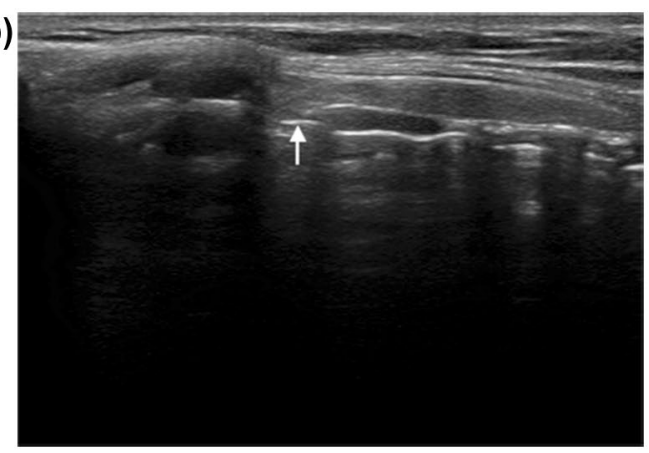

\section{How else can PoCUS be utilized for airway assessment?}

PoCUS can enhance the airway assessment through measurements of depth from the skin surface to various airway landmarks (e.g., vocal cords, suprasternal notch, thyrohyoid membrane) [1]. These measurements and their respective cut-offs appear to be more accurate for identifying potential airway difficulties than traditional bedside assessments, and are more suited to the emergency environment where patients are often unable to engage in evaluation [1]. Furthermore, where subglottic stenosis is suspected (e.g., acquired from prior intubation, congenital), measurements of the inner diameter from the level of the cricoid downward to the narrowest point are highly accurate [1]. This is performed by visualizing the subglottic space in the transverse orientation and sliding caudally to visualize the narrowest area. In pediatrics, this same approach using the inner diameter at the level of the cricoid cartilage has been shown to be superior to other methods for selecting ETT size [1].

\section{Case resolution}

Using PoCUS, you are immediately able to confirm a properly located ETT. However, you subsequently assess for the ETT depth using lung sliding and determine that this was a right mainstem intubation. You withdraw the ETT slightly and identify bilateral lung sliding, confirming correct ETT depth. After several more rounds of cardiopulmonary resuscitation, the patient achieves a return of spontaneous circulation and is transferred to the intensive care unit.

Funding None.

\section{Compliance with ethical standards}

Conflict of interest The authors declare that they have no conflict of interest.

\section{References}

1. Gottlieb M, Holladay D, Burns KM, Nakitende D, Bailitz J. Ultrasound for airway management: an evidence-based review for the emergency clinician. Am J Emerg Med. 2020;38(5):1007-13.

2. Gottlieb M, Holladay D, Peksa GD. Ultrasonography for the confirmation of endotracheal tube intubation: a systematic review and meta-analysis. Ann Emerg Med. 2018;72(6):627-36.

3. Grmec S. Comparison of three different methods to confirm tracheal tube placement in emergency intubation. Intensive Care Med. 2002;28(6):701-4.

4. Gottlieb M, Alerhand S, Long B. Ultrasound for intubation confirmation of COVID-19 patients. West J Emerg Med. 2020. https ://doi.org/10.5811/westjem.2020.7.48657.

5. Tessaro MO, Salant EP, Arroyo AC, Haines LE, Dickman E. Tracheal rapid ultrasound saline test (T.R.U.S.T.) for confirming correct endotracheal tube depth in children. Resuscitation. 2015;89:8-12.

6. Arthurs L, Erdelyi S, Kim DJ. The effect of patient positioning on ultrasound landmarking for cricothyrotomy. Can J Anaesth. 2020. https://doi.org/10.1007/s12630-020-01826-x (Epub ahead of print). 